\title{
Thermally Induced Wear of Ceramics
}

\author{
H.S.C. Metselaar \\ University of Twente, Twente Institute of Mechanics \\ P.O. Box 217, 7500 AE Enschede, The Netherlands \\ phone +31-(0)53-4892476, email h.metselaar@wb.utwente.nl
}

\section{Introduction}

Ceramics have a high strength and are chemically stable materials, however they are often not used because extreme wear is feared due to their brittle nature. Therefore a model has been derived, predicting the transition of mild to severe wear due to thermally induced stresses (Metselaar et al. 1999): The temperature rise due to frictional heating in a tribological contact induces thermal stresses in the material. When this stress exceeds a threshold value, fracture will occur. The severity of the contact can be described as:

$$
T S=\frac{f W V}{a K_{e f f} \Delta T_{S}}
$$

in which $\Delta T_{S}$ is the thermal shock resistance, proportional with $\frac{(1-v) K_{I c}}{E \alpha \sqrt{\pi d}}$ and

$K_{\text {eff }}=2.667\left(K_{2}+\sqrt{K_{1}^{2}+0.4 \operatorname{Va}_{1} c_{1} K_{1}}\right)$.Severe wear will occur when TS exceeds a certain threshold value that is to be determined. The validation of this model will be described here, as well as the determination of the threshold value.

\section{Validation}

In order to validate this model, wear measurements have been performed on a pin-on-disc tribometer. As disc material a zirconia was used because of its low thermal conductivity, allowing high TS-values at moderate loads and speeds. As pin materials several other ceramics were used, viz. alumina, silicon carbide, silicon nitride and zirconia. For each experiment the TS-value was calculated from the experimental conditions and the resulting coefficient of friction. These TS can be plotted against the resulting specific wear rate (in $\mathrm{mm}^{3} / \mathrm{Nm}$ ), to obtain the following graph.

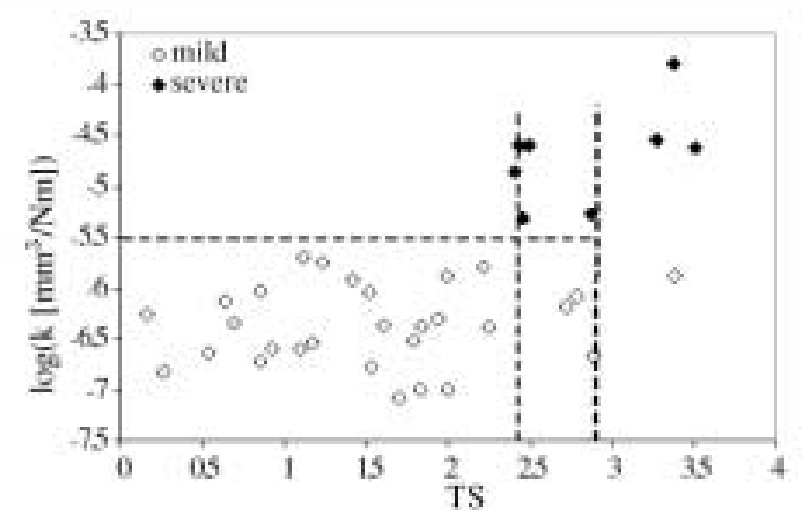

Figure 1: Overview of results of all present experiments.

\section{Conclusion}

Simple pin-on-disc experiments using different materials were performed to validate a model describing the onset of severe wear in ceramic tribosystems. From these experiments also a threshold value could be determined.

\section{References}

Metselaar, H. S. C., Winnubst, A. J. A. \& Schipper, D. J. (1999), 'Thermally induced wear of ceramics', Wear 225-229, 857-861. 